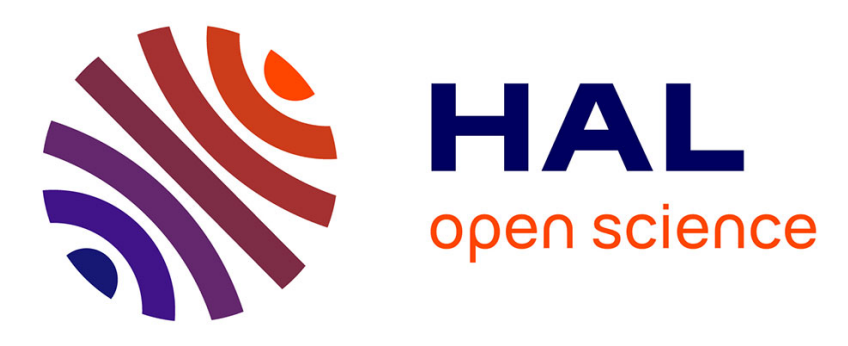

\title{
Integration of Biologically Inspired Temporal Mechanisms into a Cortical Framework for Sequence Processing
}

\author{
Hervé Frezza-Buet, Nicolas P. Rougier, Frédéric Alexandre
}

\section{- To cite this version:}

Hervé Frezza-Buet, Nicolas P. Rougier, Frédéric Alexandre. Integration of Biologically Inspired Temporal Mechanisms into a Cortical Framework for Sequence Processing. none. Neural, Symbolic and Reinforcement Methods for Sequence Learning, Springer, 28 p, 2000. inria-00107857

\section{HAL Id: inria-00107857 \\ https://hal.inria.fr/inria-00107857}

Submitted on 19 Oct 2006

HAL is a multi-disciplinary open access archive for the deposit and dissemination of scientific research documents, whether they are published or not. The documents may come from teaching and research institutions in France or abroad, or from public or private research centers.
L'archive ouverte pluridisciplinaire HAL, est destinée au dépôt et à la diffusion de documents scientifiques de niveau recherche, publiés ou non, émanant des établissements d'enseignement et de recherche français ou étrangers, des laboratoires publics ou privés. 


\title{
Integration of Biologically Inspired Temporal Mechanisms into a Cortical Framework for Sequence Processing
}

\author{
Hervé Frezza-Buet ${ }^{1}$, Nicolas Rougier ${ }^{2}$, and Frédéric Alexandre ${ }^{2}$ \\ ${ }^{1}$ Supélec, ${ }^{2}$ Loria, \\ France
}

\section{Introduction}

Time is an important dimension in many real-world problems. This is particularly true for behavioral tasks where the temporal factor is critical. Consider for example the analysis of a perceptual scene or the organization of behavior in a planning task. Temporal problems are often solved using temporal techniques like Markovian Models or Dynamic Time Warping. Classical connectionist models are powerful for pattern matching tasks but exhibit some weaknesses in dealing with dynamic tasks involving the temporal dimension. Thus, they are efficient for off-line statistical data processing, but must be adapted for situated tasks which are intrinsically temporal.

This adaptation can correspond to the coupling of connectionist models with classical temporal techniques, thus yielding hybrid models (Sun and Alexandre 1997). It can also correspond to the design of new connectionist architectures with specific abilities for temporal processing. We have proposed in the past a classification for temporal connectionist architectures (Durand and Alexandre 1996). Following this classification, these networks can be described according to the way time is represented within the architecture.

Architectures with an external representation of time correspond to classical neural networks like multilayer perceptrons in which only the input space is modified to include the temporal dimension and some specific mechanisms are added. The Time Delay Neural Network (TDNN) is a typical example of this kind of system. This model was introduced by (Waibel et al. 1989) to learn and recognize phonemes in automatic speech recognition tasks. The input space is a window on a spectral representation of speech with frequency and time axes, and a mechanism of weight sharing ensures invariance of position in time. Apart from that, the general architecture of a TDNN is a classical multilayer perceptron learning with a backpropagation algorithm. Models with such an external representation suffer from serious limitations because time is considered as a dimension similar to other spatial or physical dimensions.

Architectures with an internal representation of time are specific architectures designed for the purpose of dynamic information processing and are thus not classical architectures with an adapted input space. The internal representa- 
tion of time in these architectures can be built either in an implicit or an explicit way.

Concerning the implicit method, typical architectures are recurrent neural networks (Pearlmutter 1990), that manage time via the succession of their internal steady states. In this case, the current output is obtained as a function of the current input state and of the context, obtained from a copy of the previous internal state of the network (Elman 1990). This representation is called implicit because the succession of events only appears implicitly, through the context layer.

On the contrary, the explicit method for the internal representation of time clearly implements time through sequences of events that can be detected directly inside the network. Close to Markovian principles, these architectures include units that represent events, and links that represent transition probabilities between these events, thus yielding explicit sequence representations within the network. Models using this strategy of representation are often (but not always (Béroule 1990)) inspired by cortical functioning, either for memory mechanisms (Ans et al 1994) or for more general sensorimotor tasks (Alexandre 1996). Here the main problem is to design learning rules for establishing these temporal links and to integrate the latter with other classical spatial links.

The biological validity of these various strategies for time representation inside neural networks has been discussed elsewhere (Durand and Alexandre 1996). We just mention here that the external strategy could correspond to the processing of temporal sensory input like auditory input (Suga 1990), the internal implicit strategy could correspond to the highest levels of time integration (Dominey et al. 1995), (Elman 1990) and the internal explicit strategy could correspond to some biological models of cortical functioning (Burnod 1989). Many neurobiologically plausible mechanisms of time processing have been proposed in the past. They are generally using an internal representation of time. Some of the most typical will be reviewed in section 2 .

Beyond this local view on specific temporal mechanisms, the goal of this chapter is to emphasize the idea of integrating these mechanisms into a more general framework, allowing a better exploitation and articulation between them. As will be discussed in section 3, the cerebral cortex may be seen as a set of functionally and architecturally different modules, each devoted to specific aspects of time processing. Building modular architectures gathering and coupling these abilities can undoubtedly offer new temporal properties, particularly if integrated behavioral tasks are investigated, as reported in section 4 .

\section{Temporal mechanisms in biological modeling}

Even if the whole neural network domain often draws (more or less tightly) on biological inspiration, mechanisms like activation functions or learning rules are often designed with no reference to time, whereas a real neuron is a dynamic system that evolves over time. In this section, some biologically inspired temporal models of neurons available in the literature will be presented. All of them have 
been designed in order to model experimental data from conditioning paradigms involving stimulus associations, sequence management, or the ability to keep cues in memory during a delay period. Underlying temporal mechanisms will be described here and related to the functioning and to the learning of neurons. The experimental framework and the fitting of the models to the data will not be mentioned and the reader might refer to cited papers for further information concerning those points.

\subsection{Functioning mechanisms}

In simple terms, neuronal functioning can be explained as follows. Dendrites receive signals from other neurons and transmit them to the soma and the axon. Then, a non-linear functioning causes an output signal to be propagated on the axon toward other connected neurons. This signal constitutes a spike train. Only the quantity and the timing of spikes code the information. In classical neuronal models, a continuous value, the activation, stands for the mean frequency of the firing rate and thus hides individual neuronal temporal behavior. On the contrary, it is exploited by some more biologically inspired models.

Spiking neurons One of the lowest levels of description of neuronal temporal behavior is the spike itself (Gerstner 1998). In the so-called spiking neuron approach, neuronal activity is fully reported with, for each neuron $i$, the set of its firing times.

$$
F_{i}=\left\{t_{i}^{(1)}, \cdots, t_{i}^{(n)}\right\}
$$

Based on these elementary data, various neuronal operations can be implemented (Gerstner 1998). They can investigate rate coding (with an average over time, over several cycles or over a population of neurons) or pulse coding (strategies of coding based on synchronicity of spike timing).

Several models of neurons, from the simplest to the most complex (e.g. compartmental), have also been designed within this formalism (Maass and Bishop 1998). For example, the Spike Response Model describes the neuronal state $u_{i}(t)$ at time $t$ (which can be interpreted as the cell membrane potential) as the sum of the neuronal response to its own spikes (refractory period with negative kernel $\eta_{i}(s)$ ) and the response to presynaptic spikes (excitatory (resp. inhibitory) postsynaptic potential given by a positive (resp. negative) kernel $\epsilon_{i j}(s)$ ), as written in equation 2.

$$
u_{i}(t)=\sum_{t_{i}^{(f)} \in F_{i}} \eta_{i}\left(t-t_{i}^{(f)}\right)+\sum_{j \in \Gamma_{i}} \sum_{t_{j}^{(f)} \in F_{j}} w_{i j} \epsilon_{i j}\left(t-t_{j}^{(f)}\right)
$$

where $\Gamma_{i}$ is the set of neurons connected to neuron $i$ and $w_{i j}$ corresponds to the synaptic strength of the connection between the neurons $i$ and $j$.

This formalism has the advantage of being established at a very low (and thus precise) level of time. It has also given rise to a variety of theoretical as well as applied studies (Maass and Bishop 1998) which now establish spiking neurons as 
a full domain of research. However, the corresponding drawback of this formalism is that its low level of granularity implies a large amount of computation even for a simple network of neurons. Another drawback is related to the lack of well established learning rules at this level of description. That is one reason why it is important to keep in mind other formalisms describing these (and other related) temporal mechanisms.

The leaky integrator At a higher level of description than the spike, explicit temporal functions can also be used to obtain a neuronal temporal behavior. In the simple leaky integrator model (SLI), the input to a neuron at time t, denoted by $I(t)$, can take a continuous value between zero and one (Reiss and Taylor 1991). Then, the membrane potential $A(t)$ is written as:

$$
A(t+1)=f(I) \cdot I(t)+(1-f(I)) \cdot A(t)
$$

where the function $f(I)$ is defined, with constants $a$ and $d$ :

$$
f(I)=d(1-I)+a I .
$$

Finally, the output of the neuron is computed via the Heaviside function $H$ as:

$$
\operatorname{Out}(t+1)=H(A(t)-0.5)
$$

As illustrated in figure 1 , functions $A$ and $f$ are such that they provide to the neuronal internal state a wave input attack in the time $1 / \ln (a)$ and a wave input decay in the time $1 / \ln (d)$. It is thus possible to determine the shape of the activity with the choice of constants $a$ and $d$. Of course, these phenomena can also be obtained within the spiking neuron formalism (Gerstner 1998), but at a much higher computational cost.

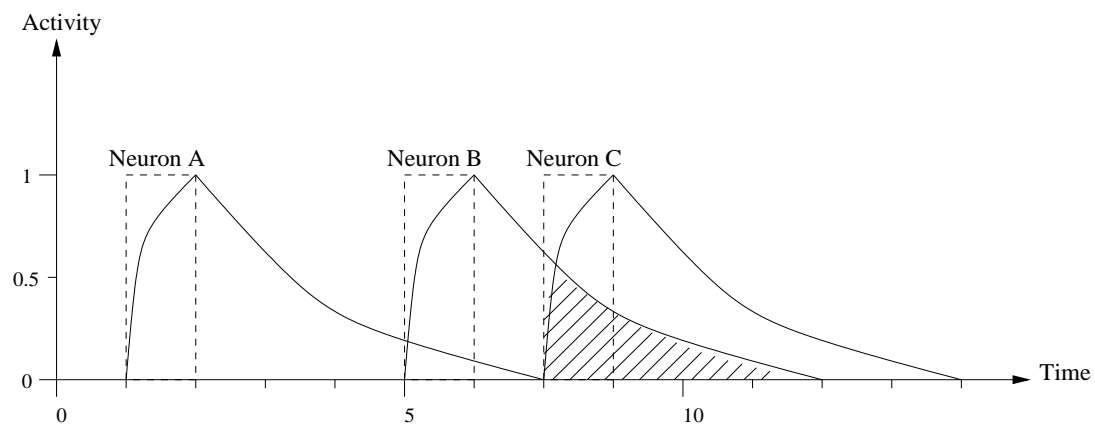

Fig. 1. This figure represents the typical shape of activity obtained with a trace mechanism. It also illustrates how this mechanism enables two events occurring at distinct instants to have lasting activities, to meet and to interact via learning 
This trace mechanism, describing neurons as leaky integrators, makes the neuronal activity last longer than its input. We will explain below how this mechanism is important for a network to properly learn and recall sequences.

The gated dipole Synaptic functioning has also been studied, with differential equations over time describing at each time the variation of some state variables, as in the SLI model. This approach has led to many models, sharing common features. These features are described below, on the basis of the gated dipole model introduced by Grossberg (Grossberg 1984). The model is a good illustration of the kind of complex temporal behavior that can arise from straightforward equations.

The gated dipole is grounded on the modeling of synaptic dynamics. The role of the synapse is to transfer a signal from presynaptic to postsynaptic nervous fibers. Let $t$ be the (continuous) time parameter, $S(t)$ the value of the presynaptic activity, $T(t)$ the value of the postsynaptic activity and $z(t)$ the conductance of the synapse provided by chemical neurotransmitters. Equation 6 describes the way $S$ is transmitted through the synapse over time, figure 2 illustrates it.

$$
T(t)=S(t) . z(t)
$$

A perfect conduction would correspond to a constant conductance $z(t)$ over time. When $S(t)$ occurs (becomes non null), some previously stored neurotransmitters are emitted to provide conductance (this is considered as instantaneous in the model). The synapse continuously produces neurotransmitters to "refill" the stock until saturation. The production and consumption of neurotransmitters can then be described with equation 7 .

$$
\frac{d}{d t} z(t)=A \cdot(B-z(t))-S(t) \cdot z(t)
$$

The first term of equation 7 represents the production of neurotransmitters, with a speed $A$, until the level $B$. The second term is the consumption of neurotransmitters by the signal $S(t)$. If signal $S(t)$ is kept constant, production and consummation of neurotransmitters complement each other and the output signal $T(t)$ reaches an equilibrium value such that:

$$
\begin{array}{r}
\quad \frac{d}{d t} z(t)=0=A .(B-z(t))-S(t) \cdot z(t) \\
\Rightarrow z(t)=\frac{A B}{A+S(t)} \Rightarrow T(t)=\frac{A B \cdot S(t)}{A+S(t)}
\end{array}
$$

The function $T=f(S)$ at the equilibrium state is non-linear, monotonic increasing and saturates with the value $A B$. Such a function is similar to the sigmoidal transfer function used with the classical formal neuron. Outside the equilibrium case, the dynamics of the synapse, shown in figure 2, have interesting temporal properties. For example, overshoots and undershoots can trigger events when $S(t)$ respectively sets and resets. Moreover, the decay from overshoot to habituation can be considered as a progressively decaying trace of the burst of $S$. The 
concept of trace is useful to correlate time separated events, as will be discussed later.

This model is suitable for detecting transitions of signals and illustrates well the kind of temporal properties easily obtained by using appropriate intrinsically temporal models.
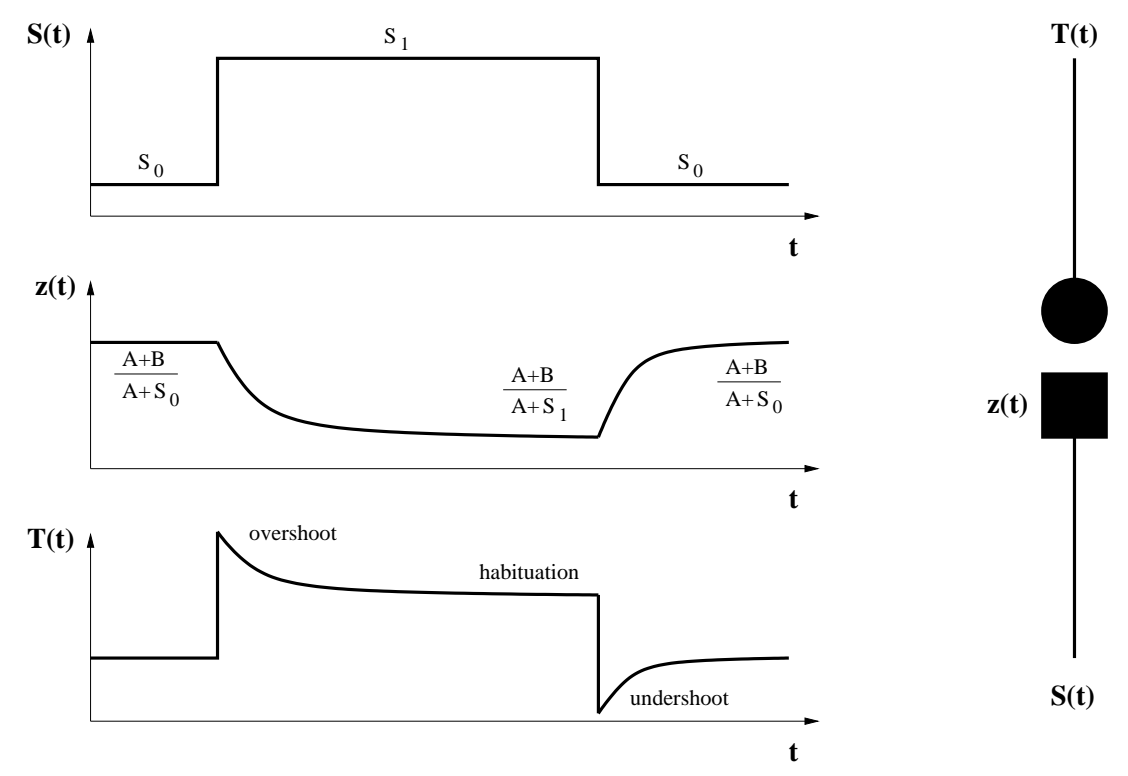

Fig. 2. The gated dipole, from (Grossberg 1984).

\subsection{Learning mechanisms}

Classical static learning mechanisms are often based on Hebbian rules, which compute weight variations from the correlation between presynaptic and postsynaptic activities, according to the rule:

$$
\Delta W_{i j}=\alpha S_{i} \cdot T_{j}
$$

This static view is obviously an approximation since it is clear that, as a consequence of presynaptic activity, postsynaptic activity is not simultaneous but consecutive to presynaptic activity. Several temporal learning mechanisms have been proposed and take this constraint into account.

The common feature that underlies these mechanisms is the use of traces, so that instantaneous correlations between traces of signals signify temporal correlations of those signals (cf. figure 1). The shapes of the traces, and the properties of the learning rules, endow the models with their own specific properties. 
Learning the earliest predictor Adaptive behavior has been widely studied throughout classical (Pavlov 1927) and instrumental (Skinner 1938) conditioning. The classical conditioning experiment is described in the following terms in (Sutton and Barto 1981):

..., the subject is repeatedly presented with a neutral conditioned stimulus, that is, a stimulus that does not cause a response other than orienting responses, followed by an unconditioned stimulus (UCS), which reflexively causes an unconditioned response (UCR). After a number of such pairings of the CS and the UCS-UCR, the CS comes to elicit a response of its own, the conditioned response (CR), which closely resembles the UCR or some part of it.

There exist several variations of this simple experiment. For example, one can try to condition the subject with the help of several $C S_{i}$. The point is that the delay separating $C S_{i}$ from $U C S$ (inter-stimulus interval or $I S I$ ) plays a crucial role in the success or failure of the conditioning. Neural modeling takes this point into account. Furthermore, a large amount of experimental paradigms (overshadowing, blocking, etc.) give serious clues concerning the temporal mechanisms underlying conditioning.

Sutton and Barto (Sutton and Barto 1981) propose a model based on activity traces which is able to take these kinds of data into account. The model is grounded in a formal neuron (cf. figure 3) where input $x_{0}$ is the value of the $U C S$, other inputs $x_{i}$ are respective values of the $C S_{i}$ and $y$ is indifferently the $C R$ or the $U C R$.

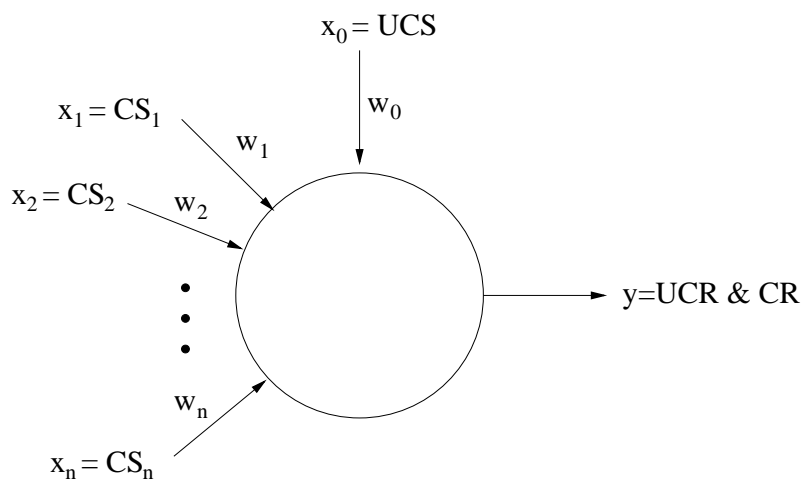

Fig. 3. The adaptive element of (Sutton and Barto 1981).

The model is driven by the following equations, where $f$ is a sigmoid function, $\alpha$ and $\beta$ are positive constants with $0 \leq \alpha, \beta<1$ and $c$ is a positive constant determining the learning rate: 


$$
\begin{aligned}
\bar{x}_{i}(t+1) & =\alpha \bar{x}_{i}(t)+x_{i}(t) \\
\bar{y}(t+1) & =\beta \bar{y}(t)+(1-\beta) y(t) \\
y(t) & =f\left[\sum_{j=1}^{n} w_{j}(t) x_{j}(t)\right] \\
\forall i \in[1 . . n], w_{i}(t+1) & =w_{i}(t)+c[y(t)-\bar{y}(t)] \bar{x}_{i}(t)
\end{aligned}
$$

Each input $x_{i}$ and $y$ has a trace activity, respectively $\bar{x}_{i}$ and $\bar{y}$. At the beginning of conditioning, in the case of a single $C S$ (corresponding to a single input $x$ ), the output $y$ is activated at the same time as UCS (or $x_{0}$ ) because of the fixed weight $w_{0}$. Meanwhile, if $\bar{x}$ is active, it will increase its weight $w$ since this latter is proportional to $(y(t)-\bar{y}(t))$ and $\bar{x}$. It will then soon be able to trigger the output on its own. The adaptive element has learned to trigger a response whenever CS is present just before UCS. As in the classical conditioning paradigm, the delay separating the onset of $C S$ and $U C S$ plays a crucial role since learning only occurs in case of temporal overlap between a positive trace of $x$ and a "burst" of $(y(t)-\bar{y}(t))$.

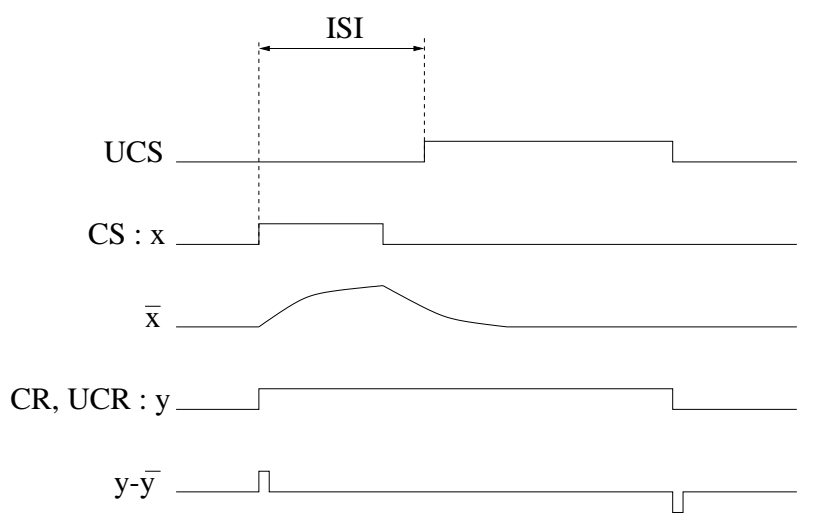

Fig. 4. Time courses of element variables after conditioning. CS elicits a response of its own.

Another aspect of classical conditioning is the context factor, which may be determinant during the learning phase. For example, when a first association $C S_{1} \rightarrow C R$ has been learned, the addition of a new $C S_{2}$ contingent to $C S_{1}$ generally implies a very weak $C S_{2} \rightarrow C R$ association or no association at all. This phenomenon is called "blocking" and is modeled by the Rescorla-Wagner theory (Rescorla and Wagner 1972) which states that organisms only learn when 
events violate their expectations. Figure 5 shows the evolution of weights for such a blocking paradigm, where three phases can be distinguished:

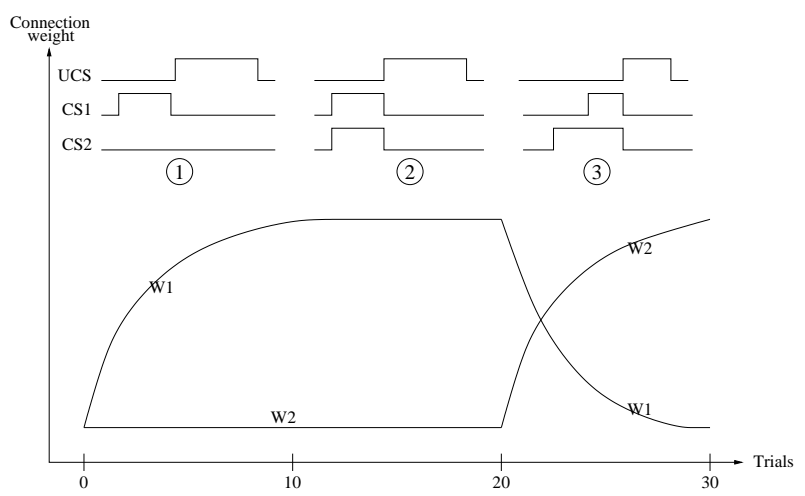

Fig. 5. The blocking paradigm. As long as $C S_{2}$ is not the earliest predictor (step 1 and 2 ), $w_{2}$ remains unchanged. As soon as $C S_{2}$ becomes the earliest predictor (step 3), $w_{1}$ decreases to zero while $w_{2}$ reaches its asymptotic value.

1. This is a classical conditioning experiment with only one $C S_{1}$. Weight $w_{1}$ is then increased up to its asymptotic value.

2. A second $C S_{2}$ contingent to $C S_{1}$ is added, but, since learning has occurred at a previous phase, output (triggered by $C S_{1}$ ) onset is now overlapping with $C S_{1}$ onset. $C S_{2}$ is then unable to learn anything since its trace activity $\bar{x}_{2}$ is not positive during the output onset. Weights $w_{1}$ and $w_{2}$ remain the same.

3. $C S_{2}$ onset is now earlier than $C S_{1}$, trace activity $\bar{x}_{2}$ is now positive during the output onset (triggered by $C S_{1}$ ), weight $w_{2}$ is then increased. The consequence is that output will be triggered sooner and sooner by $C S_{2}$ up to the point where $\bar{x}_{1}$ will be positive during the output offset. Then $w_{1}$ will be decreased to zero. $C S_{2}$ has become a better predictor of $U C S$ than $C S_{1}$.

Learning the date of the predictor Learning the earliest predictor is useful for anticipating the consequence of an event. However, if the time interval that separates the event and its consequence is nearly constant, it may be useful to be ready only when the consequence occurs. A mechanism allowing the memorization of this time interval has been proposed in (Grossberg and Schmajuk 1987), (Grossberg and Schmajuk 1989). This mechanism is grounded on a trace that arises after the burst of a signal and then shuts down. This defines a time interval, after the burst of the signal, and correlation can be computed during this interval. The idea is then to provide many synapses with different time constants, and thus to allow an overlap during complementary intervals. The model is called the Spectral Timing Model. 
Let us first describe the functioning of one synapse, and then the use of a range of such synapses for anticipation. Let $I_{C S}$ be a conditioned signal (a step function), and $I_{U C S}$ be the unconditioned signal. When learning has occurred, $I_{U C S}$ has to be anticipated when it should occur, according to $I_{C S}$. Synapse $i$ is described by three variables, according to the following equations, where $f$ is a sigmoidal function.

$$
\begin{aligned}
\frac{d}{d t} x_{i} & =\alpha_{i}\left[-A x_{i}+\left(1-B x_{i}\right) I_{C S}\right] \\
\frac{d}{d t} y_{i} & =C\left(1-y_{i}\right)-D f\left(x_{i}\right) \\
\frac{d}{d t} z_{i} & =E f\left(x_{i}\right) y_{i}\left[-z_{i}+I_{U C S}\right]
\end{aligned}
$$

Equation 14 allows $x_{i}$ to transmit $I_{C S}$ with a delay, depending on parameters. Equation 15 describes spontaneous production of neurotransmitter $y_{i}$ and consumption of this neurotransmitter when the synapse transmits $x_{i}$. This equation is similar to equation 7 . As a delayed signal $x_{i}$ is transmitted through the synapse, using neurotransmitter $y_{i}$, the transmitted value of $x_{i}$, i.e the product $f\left(x_{i}\right) y_{i}$, is a trace of the occurrence of $I_{C S}$. Then, equation 16 describing the strength of association $z_{i}$ performs the evolution of $z_{i}$ toward $I_{U C S}$, but only when the trace of $I_{C S}$ is strong enough. The shape of the traces for a high $\alpha_{i}$ (in plain line) and for low $\alpha_{i}$ (in dashed line) is illustrated in figure 6.
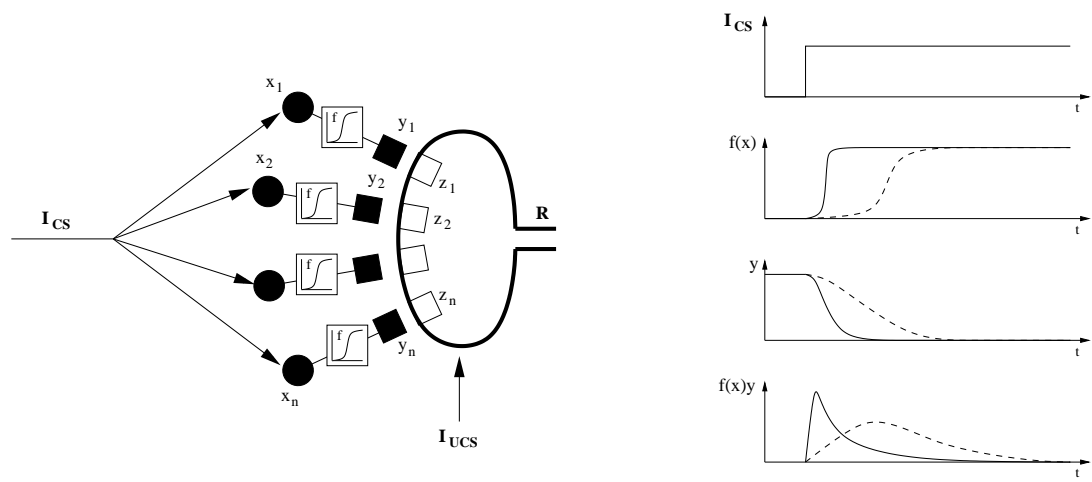

Fig. 6. Spectral Timing Model, from (Grossberg and Schmajuk 1987).

Spectral timing consists then in endowing a neuron with a battery of synapses, having different values $\alpha_{i}$, for correlations with a given $I_{C S}$ (cf. figure 6 ). The 
response of the neuron is then given by equation 17 .

$$
R=\left[\sum_{i} f\left(x_{i}\right) y_{i} \cdot z_{i}-F\right]^{+} \text {with } \begin{cases}x^{+}=x & \text { if } x \geq 0 \\ x^{+}=0 & \text { otherwise }\end{cases}
$$

The behavior of the neuron then consists in responding to an $I_{C S}$ by summing traces that have been associated with $I_{U C S}$ (they have a high $z_{i}$ value). The response then occurs at the moment when $I_{U C S}$ should occur.

Finally, let us mention that the spectral timing paradigm has also been successfully implemented with a range of leaky integrators as a model of frontal cortex (Dominey et al. 1995).

Context-dependent learning The models previously discussed were grounded on classical conditioning experiments, and deal with the association of two signals. Some other models describe a synapse concerned with a third signal, modulating the associative role of the synapse. This defines the concept of the synaptic triad.

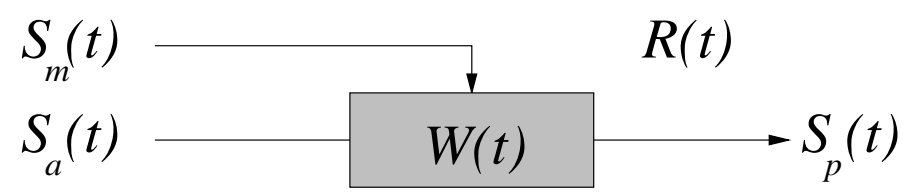

Fig. 7. The synaptic triad, from (Dehaene and Changeux 1989).

The synaptic triad model of Dehaene and Changeux (Dehaene and Changeux 1989), illustrated in figure 7 , involves a presynaptic activity $S_{a}(t)$, a postsynaptic activity $S_{p}(t)$, and a modulation signal $S_{m}(t)$ that acts on the synaptic weight $W(t)$. The weight $W(t)$ is a trace of the modulation activity.

$$
W(t+1)= \begin{cases}\alpha_{p} W(t)+\left(1-\alpha_{p}\right) W^{m}(t) & \text { if } S_{m}(t)>0.5 \\ \alpha_{d} W(t) & \text { if } S_{m}(t)<0.5\end{cases}
$$

The $\alpha_{p}$ and $\alpha_{d}$ parameters are in the range $[0,1]$. The weight $W(t)$ is a trace that increases toward the current maximal authorized weight $W^{m}(t)$ when the modulation signal $S_{m}(t)$ is high, and decreases otherwise. The contribution of all the synapses $i$ to the output signal $S_{p}(t)$ is given by:

$$
S_{p}(t+1)=\sum_{i} W^{i}(t) S_{a}^{i}(t)
$$

The temporal learning rule for the triad actually computes the value $W^{m}$ of the synapse. The learning rule is given by:

$$
\delta W^{m}(t)=\beta R(t) \frac{W(t)}{W^{m}(t)} S_{a}(t)\left(2 S_{p}(t)-1\right)
$$


The function $R(t)$ is a reinforcement signal, related to the experimental framework of the model (Dehaene and Changeux 1989). It will be considered as a positive constant and will not be discussed here. The maximal weight $W^{m}$ increases according to the cooccurence of presynaptic signal $S_{a}$ and postsynaptic signal $S_{p}$, as for Hebbian learning rules, but this occurs only when the trace $W$ of $S_{m}$ is close to its maximum value. The signal $S_{m}$ can be viewed as a context value, and the synapse will conduct signals only when the context is active (cf. equation 19).

More complex contextual rules have been developed since the initial model by Dehaene and Changeux, dealing with non-simultaneity of presynaptic and postsynaptic signals, i.e one has to come before the other when context is active in order to increase the weight of the synapse (see (Guigon 1993) for example).

\subsection{Biological temporal mechanisms}

This overview of some functioning and neuronal learning mechanisms illustrates the variety of temporal neuronal properties that can be exploited in artificial neural networks (and that are absent in classical static models). As mentioned above, these mechanisms are generally used to model experimental data and often stick to the experimental framework. They have to be integrated in a more complete architecture in order to address wider range of real-world problems. For example, this has been successfully done for radar imaging by Grossberg and colleagues (Gove et al. 1995), (Grossberg et al. 1995).

Engineering-like applications, like autonomous robot control, are often tackled by Markov Decision Processes such as Q-Learning (Watkins 1989) (see (Littman 1996) for a good overview of these techniques). In the latter case, time is considered as a discrete parameter, ordering a series of synchronous interactions between the agent and the external world. However, this kind of approach seems to be seldom applied to real asynchronous problems.

Biological models may be an efficient way to overcome this difficulty, due to the robustness of temporal mechanisms like the trace concept. Our claim is that the integration within a cortical framework of such elementary biologicallyinspired mechanisms can lead to efficient systems for real-world applications.

\section{Cortical modeling}

The goal of establishing a framework for cortical modeling is to bridge the gap between the isolated temporal mechanisms and the distributed and polymodal nature of the cerebral cortex itself. This makes it possible to efficiently express complex behaviors, including several temporal resolutions. Before proposing (in the next section) our computational models for this framework, we report the outline of the biological model that underlies it. The reader can refer to the original description of the model (Burnod 1989) for more details about the underlying biological data. These data yield two levels of description. First, at the global level, architectural and functioning data give hints about different kinds 
of processing regions in the cortex, and information pathways between them. Second, at a more local level, neuronal circuitry is described together with the corresponding functioning and learning rules.

\subsection{The global level: a network of areas}

The global description of the four major lobes (frontal, parietal, temporal and occipital) with the functional description of the cortex proposed by Brodmann as early as 1909 (Brodmann 1909), gives precious hints for a functional approach. It makes the distinction of four major types of areas, as illustrated in figure 8 and described below (using the formalism of this figure).

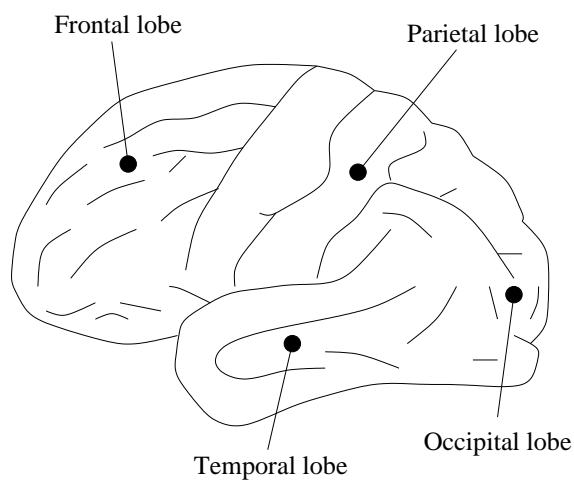

(a) Main lobes of the cerebral cortex

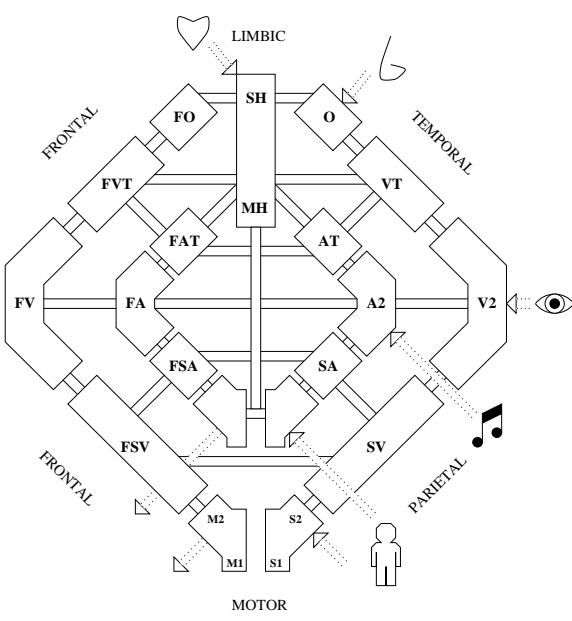

(b) Model of the connectivity between cortical areas (adapted from (Burnod 1989))

Fig. 8. Anatomical and functional view of the cerebral cortex

Sensory areas These mono-modal areas map the sensory information coming from the different body receptors which can be divided into five main sensory poles (auditory (A), visual (V), somesthesic (S), olfactory (O) and internal molecular ( $\mathrm{SH})$ ). Moreover, the information flow is structured in such a way that topology is conserved from peripheral receptors to cortical sensory areas. A fixed mono-modal perceptive sequence can be encoded at this level. 
Motor areas (M) These mono-modal areas allow the performing of actions upon the internal world (e.g. hormonal secretion) or the external world (e.g hand movement). Actions can be executed simultaneously but also coordinated in a complex sequence of movements (but fixed at this level).

Posterior associative areas These polymodal areas, including temporal and parietal areas, are crucial to cortical organization since they allow the linking of at least two areas, one with the other. They will for instance allow direct sensorimotor coordination encoding in the case of a link between a sensory and a motor area (e.g. hand-eye coordination). Moreover, these associative areas may also link two sensory areas or two other associative areas, allowing in this way the construction of a more structured and integrated representation of information. Stereotypical sensorimotor sequences (e.g. reaching one's mouth with one's hand) can be learned in these areas.

Prefrontal associative areas From a functional point of view, prefrontal associative areas have to be distinguished from their posterior counterparts. The former are generally action-oriented and play a major role in temporal organization of behavior. Furthermore, the privileged relations with the posterior cortex (cf. fig.8(b)) and the presence of specific temporal mechanisms within prefrontal units (bistable) make prefrontal areas able to construct and coordinate dynamic temporal sequences grounded on posterior ones (e.g. guidance of the hand toward a goal in the focus of attention).

Information pathways To throw light on the nature of cortical organization, cortical modeling defines a framework for a distributed polymodal representation of information. The integration of numerical data into more structured and "sub-symbolic" reference frames is possible throughout a hierarchy of polymodal areas. We are thus offered a way of keeping to some extent the robustness of numerical data while manipulating this data at a higher level. These cortical mechanisms involve (mainly in the posterior cortex) statistical and slow learning, and perform a kind of extraction of the world regularities through the construction of stereotypied temporal sequences. The ability to model complex and more dynamic behaviors requires additional mechanisms that can be provided by frontal areas or even by extra-cortical structures. The cortical network is not fully interconnected but defines via associative areas three privileged pathways between the motor system, the internal state and the perception of the outside world in the following way:

- parietal areas relate the outside world with the motor system

- temporal areas relate the outside world with the internal state

- frontal areas relate the internal state with the motor system

Moreover, as shown in figure 8(b), there are privileged connections between frontal areas and posterior cortex: each posterior area is mirrored within the 
frontal lobes. This anatomical design suggests an interlaced cooperation between perceptive posterior representation and frontal motor ones, for the temporal organization of behaviour. Indeed, frontal areas are believed to play a central role in most complex temporal behaviors such as anticipation, planning, working memory or any other dynamic temporal sequencing behavior.

\subsection{The local level: neuronal assemblies}

A more detailed analysis of the inner organization of the cortical sheet may also describe this as a large set of elementary circuits: the cortical minicolumns. Each of those minicolumns receives a subset of the intra or extra-cortical information and because of the topological property of the cortical areas, neighboring minicolums will tend to receive the same subset of information. These groups of minicolumns are called maxicolumns: they share the same information subset but are able to apply different filters on it. The model of the cortical column reported in (Burnod 1989) describes the functioning and learning properties of such maxicolumns, which are different from those of the formal neuron.

Architecture The cortical minicolumn (also called cortical column) is a group of a maybe a hundred interconnected neurons where activity is essentially related to the pyramidal neurons while the other neurons, excitatory or inhibitory interneurons, mainly participate in the inner mechanism of the column. Moreover, the cortical column is a six layered structure (cf. figure 9) where layers I to III allow communication with other cortical columns while layers IV to VI allow communication with extra-cortical structures. Depending on the area the column belongs to, the size of each layer may vary greatly.
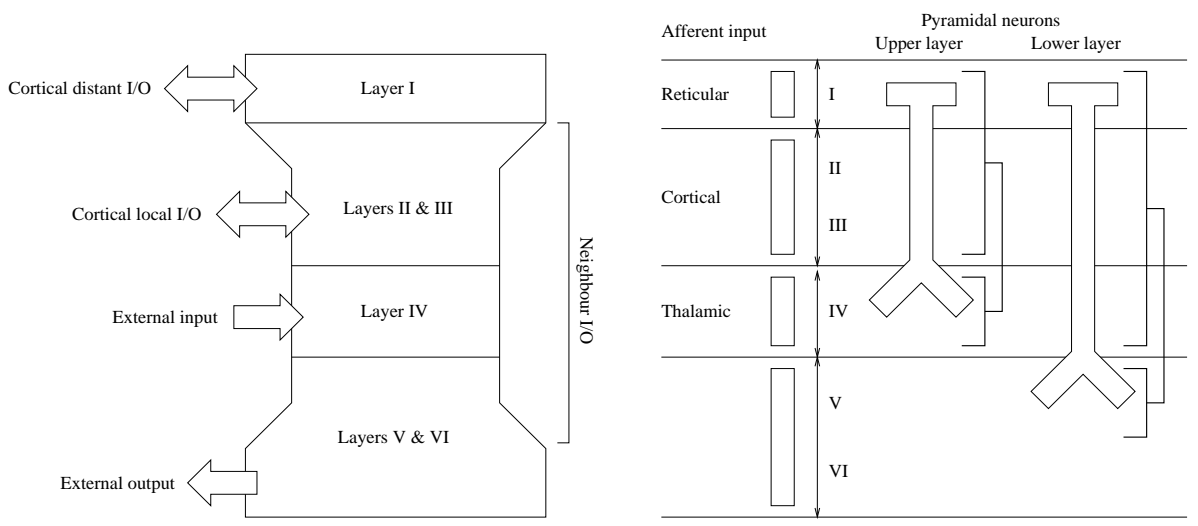

Fig. 9. The six layered structure of the cortical column and the corresponding input/output data channels 
Basic operations The inner mechanisms of the column result in three possible distinct levels of activity for the pyramidal neurons:

- Inhibited level E0 represents very weak activity

- Low level E1 represents small variations of relatively low frequencies $(5 \mathrm{~Hz}$ to $10 \mathrm{~Hz}$ ). We will refer to this priming state as the call state

- High level E2 represents a much higher frequency $(50 \mathrm{~Hz}$ to $100 \mathrm{~Hz})$. We will refer to this state as the satisfaction state

Spatial filters As said before, a maxicolumn is a set of several neighboring cortical columns sharing the same subset of information and constitutes a functional module. As long as there is no specialization of these columns, they will often be activated in a moderate way for any pattern of information. Learning is then the ability for a minicolumn to become specialized on a precise pattern of information while others in the same maxicolumn are inhibited (coupling/uncoupling). This task corresponds to filtering or feature extraction.

Spatio-temporal filters Cortical columns are able to activate themselves at three distinct levels. Activation of a column at level E2 requires the simultaneous activation of both cortical and thalamic inputs. Consequently, when a column A receives a cortical input alone or a thalamic input alone, it will not reach level E2 but rather level E1. This activity E1 is nonetheless propagated to all neighboring columns. If among them, one (C) is excited via its thalamic input, it will reach level E2 and will produce an extra-cortical action (whatever the target). This action will then modify the thalamic context, which may now be propitious for the activation of column A to level E2. Column A will then learn to preferentially call column $\mathrm{C}$ since this latter is favorable to the excitation of the former (cf. figure 10). This mechanism may be seen to some extent as a goal directed search: the level E1 is a desired or calling state, while level E2 is the satisfaction state.

The temporal mechanism of spreading intra-cortical activation indeed allows the search for sequences that are able to satisfy the calling column. Learning will then consist in slowly orienting the call activity toward columns that help to reach the satisfaction state.

Bistable units All the prefrontal area units share a common mechanism: the bistable mechanism. Bistable units possess two stable states: a resting state and a sustaining state. Both ON (at rest to sustained activity) and OFF (sustained activity to rest) transitions require an external activity (e.g. external stimuli $A$ and $B$ ) to be performed (cf. figure 11). Thus, while cortical posterior columns are only able to organize sequences at one level (e.g. A-B-C), frontal ones are able to organize hierarchical sequences (e.g. (A-(B-C))). 


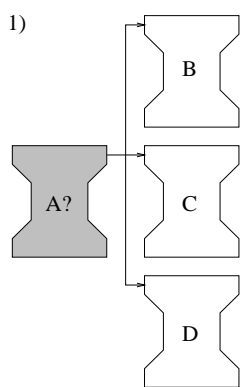

2)

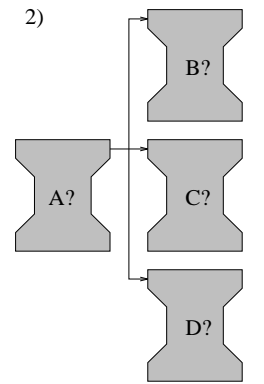

3)
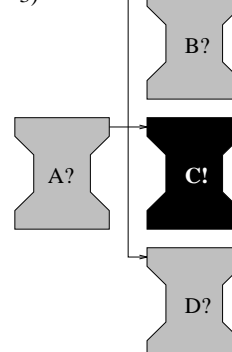
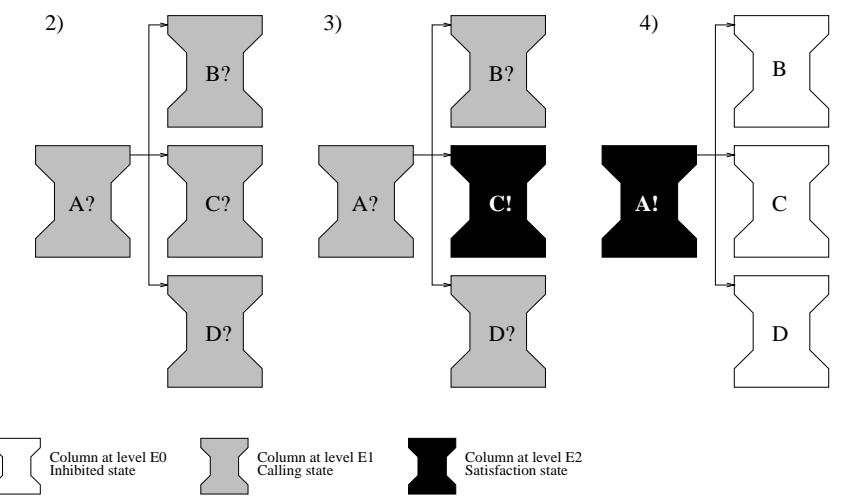

Fig. 10. 1) Column A receives cortical input and activates itself to level "E1" 2) Activity is spread to neighbouring columns 3) Thalamic context allows the activation of column C to level "E2", an action is performed that modifies the thalamic context 4) Thalamic context modification allows column A to reach activation level "E2". Learning will occur and column A will learn to preferentially call column C.

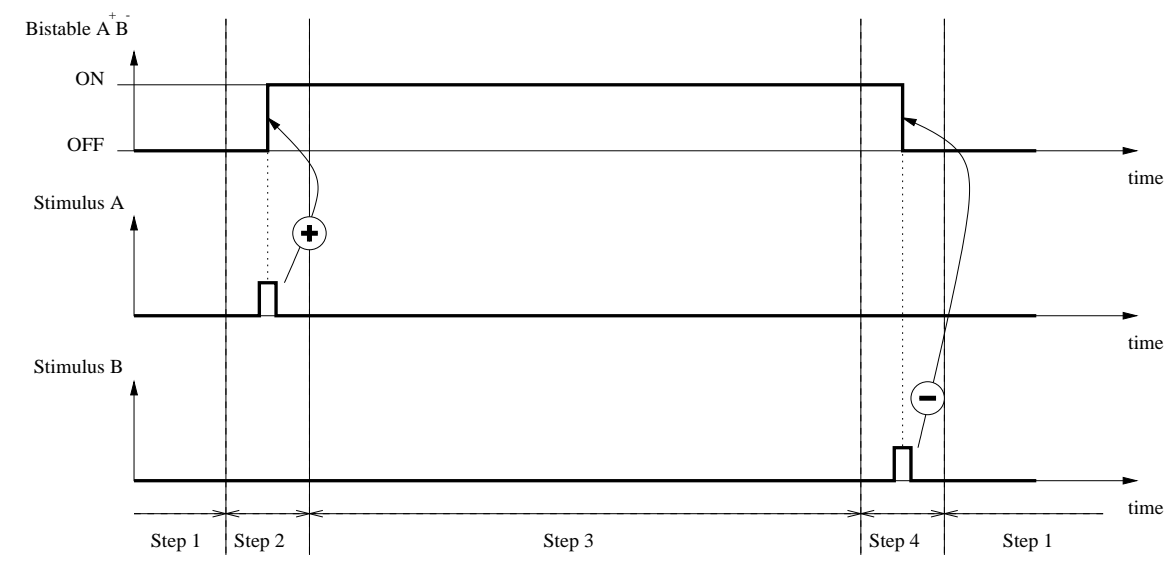

Fig. 11. 1) Bistable $A^{+} B^{-}$is at rest 2) Detection of stimulus A produces the ON transition on the bistable $A^{+} B^{-}$3) The bistable activity is sustained 4) Detection of stimulus B produces the OFF transition on the bistable $A^{+} B^{-}$ 


\section{Cortical temporal mechanisms for computer science purposes}

As computer scientists, we are specifically interested in efficient software design for real-world problems. We think that such an engineering oriented purpose can benefit from the study of biological data. Nevertheless, most biological models are not suitable for immediate software integration, but have rather been designed to explain experimental data. Classical connectionist models, like multi-layer perceptrons or Kohonen's Self Organizing Maps can be viewed as the adaptations of biological models to software engineering constraints, adding mechanisms such as backpropagation with derivable transfer functions, or explicit winner-takesall.

Concerning computation of temporal information, which is crucial when addressing real problems, a biological framework also appears to be helpful (cf. section 3 ). As is shown by the attempts we have mentioned, adaptation of biological temporal models to information processing is not obvious, and mechanisms that arise from such attempts appear to be heterogeneous (cf. section 1). We propose that one way to design reusable algorithms is to refer as precisely as possible to a cortical framework, allowing us to deal with highly integrated architectures. Integrated temporal processing implies different levels of time computation that have to be consistent one with each other. Within the same cortical paradigm, presented in section 3, we first describe low level (small time constant) mechanisms that are applied to temporal pattern recognition. We then consider causality detection, applied to the learning of higher level reactive abilities of an autonomous robot. Finally, we look at temporal mechanisms involved in neural planning with bistable units.

\subsection{Monomodal sequences within a map}

The Temporal Organization Map (TOM) architecture (Durand and Alexandre 1996) has been proposed as a model of the auditory cortex and applied to speech processing. At the lowest level, a simple cochlea model (Hartwich and Alexandre 1997) performs a spectral transformation of the speech signal. The processing level is a map of super-units, each super-unit standing for a maxicolumn, as described in section 3.2. As a first temporal mechanism, each super-unit has a spatio-temporal receptive field. The spatial field corresponds to the integration of a set of contiguous fibers from the cochlea model. The temporal field corresponds to a leaky integration, controlled by a decay parameter. This short term memory allows the integration of activation within an interval of time.

The second temporal mechanism is performed within the processing map. It corresponds to intra-map temporal links between super-units which can build explicit sequences of activation within the map. Robustness to sequence distortion (insertion or deletion) is obtained through the interaction of both temporal mechanisms. A division mechanism was also implemented and leads to specializations within super-units in order to differentiate sequences passing through the same units. 
This model was applied to spoken digit recognition using some classical benchmarks in speech processing (Durand and Alexandre 1996). It has obtained performances similar to the best stochastic models, with a better performance given the temporal and spatial complexity of the algorithm.

\subsection{Multimodal causality sequences for reactive integrated behaviour}

The mechanism described here is related to internal computation within an artificial cortical map. The maxicolumns (see section 3.2) of the map are involved in the learning of temporal regularities, and are the basic units of the model. As such, a unit represents a population of synchronous cortical columns that receive the same information. Three kinds of activation have been defined, as described for a single cortical column (Burnod 1989).

First, unit $i$ stores an excitation activity $E_{i}^{\text {exci }}$ as soon as the perceptive events $e_{i}$ it is associated with occur. Another associated recency signal $E_{i}^{\text {rec }}$ is used as a trace of the occurrence of the event. It is initialized to 1 and linearly decays over time. When it reaches 0 , the stored intensity $E_{i}^{\text {exci }}$ is reset to 0 . The use of two variables $E_{i}^{\text {exci }}$ and $E_{i}^{\text {rec }}$ prevents the confusion of strong old events with weak recent ones.

Second, a call activity of unit $i$ means that event $e_{i}$ is useful, and has to occur. For simplification here, this activity will be considered as a boolean value ( 1 or 0$)$, whereas it is, in the model, a continuous value, representing the strength of the request for $e_{i}$.

Third, when a specific event that was requested through the call activity has occured (excitation of a called unit), the unit is said to be satisfied.

Learning by specialization When learning occurs at the level of a cortical maxicolumn, columns inside the maxicolumn, that were synchronously firing, separate into two asynchronous parts. Each part can split again, further refining learning (see (Burnod 1989) for details). As units of the model represent synchronous columns, the effect of learning at the level of a unit is the creation of a new unit, both units representing the new synchronous sets of columns.

Temporal learning rule The aim of the learning rule presented here is to build sequences of units, based upon perception. Let us suppose that a given unit $i$ is called, and that the associated event $e_{i}$ always occurs after another event $e_{j}$, i.e. after $E_{j}^{\text {exci }}$ of unit $j$ is set. It is then possible to conclude that getting $e_{j}$ is a means to get the requested $e_{i}$, i.e. call activity has to spread from unit $i$ to unit $j$. If we consider unit $i$ as the goal "getting occurrence of $e_{i}$ " when it is called, unit $j$ can be viewed as a subgoal to be called. The learning rule described in equation 21 allows the detection of the goal/sub-goal relationships between units, by increasing weights between units. When a subgoal is detected, it splits, and the split unit is devoted to receiving a call activity from the goal. As it is 
called after the call activity at the level of the goal, the subgoal can play the role of a goal for other units in the map, in order to extend the causal sequence.

Let $i$ be the unit whose subgoals are detected by using the mechanism, and $j$ one of the other units in the map. The weight $w_{i j}$, initially null, represents the causal relationship between $i$ and $j$, and the splitting of $j$ occurs when it reaches 1. A flag $\delta_{i j}$ is associated with each $w_{i j}$. Weights are updated according to the procedure 21 .

$$
\begin{aligned}
& \text { if } E_{j}^{\text {rec }}=1, \delta_{i j} \leftarrow 1 . \\
& \begin{array}{ll}
\text { if } E_{i}^{\text {rec }}=1 \text { and } E_{i}^{\text {call }}=1,(\text { satisfaction of } i) \\
\text { case } E_{j}^{\text {rec }}=M^{i} \text { and } M^{i}>0: w_{i j} \leftarrow w_{i j}+\tau \cdot E_{j}^{\text {rec }} \cdot E_{j}^{\text {exci }} \\
\text { case } 0<E_{j}^{\text {rec }}<M^{i} & : w_{i j} \leftarrow w_{i j}+\tau \cdot\left(E_{j}^{\text {rec }}-M^{i}\right) . E_{j}^{\text {exci }} \\
\text { case } E_{j}^{\text {rec }}=0 & : w_{i j} \leftarrow w_{i j}-\theta \\
\text { in all cases } & : \delta_{i j} \leftarrow 0
\end{array} \\
& \text { else if } E_{i}^{\text {call }}=1 \text { and } E_{j}^{\text {rec }} \text { reaches } 0, \\
& \qquad \begin{array}{l}
\text { else nothing to compute. } \\
\text { wij }
\end{array}
\end{aligned}
$$

Parameters $\tau$ and $\tau^{\prime}$ are fixed learning rates, the symbol $\leftarrow$ stands for variable setting and $M^{i}=\max _{j} E_{j}^{\text {rec }}$. Learning $w_{i j}$ occurs only when unit $i$ is a goal $\left(E_{i}^{\text {call }}=1\right)$. When an event $e_{j}$ occurs $\left(E_{j}^{\text {rec }}=1\right)$, the flags $\delta_{k j}$ for all $k$ are raised. If the goal $i$ is satisfied $\left(E_{i}^{\text {rec }}=1\right.$ and $E_{i}^{\text {call }}=1$ ), the weight $w_{i J}$ to unit $E_{J}$ that has been excited the most recently $\left(E_{J}^{\text {rec }}=M^{i}\right)$ increases, proportional to both the recency and the stored intensity $E_{J}^{\text {exci }}$ of the event $e_{J}$. For the other $j$ units that are "quite recent" $\left(E_{j}^{\text {rec }}>0\right)$, the weights $w_{i j}$ are decreased, proportionally to the relative age $E_{j}^{\text {rec }}-M^{i}$ of $e_{j}$ occurrence, and also according to $E_{j}^{\text {exci }}$, consistent with the rule for unit $J$. The mechanism for the most recent unit $J$ and the other recent $j$ is a competition for recency, detecting the last predictor of the goal satisfaction. Events that have not occurred before the satisfaction of the goal $i\left(E_{j}^{\text {rec }}=0\right)$ are decreased with a decay value. Note that satisfaction of the goal $j$ resets the flags $\delta_{i j}$ of related weights $w_{i j}$. If an event $e_{j}$ occurs, without being followed by the satisfaction of the goal, i.e the trace $E_{j}^{\text {rec }}$ reaches 0 , if the goal is still being called $\left(E_{i}^{\text {call }}=1\right)$ and if it has not been satisfied since the occurrence of $e_{j}$ (the flag $\delta_{i j}$ is still raised), then the weight $w_{i j}$ is decreased.

This mechanism has been shown to be robust to different kinds of temporal noise, mainly distortion of sequences, insertion of events, permutation of items in the sequence (Frezza-Buet and Alexandre 1999). It is suitable for detecting sequences of causality between perceptive events that are intrinsically asynchronous, which is the case for real perception. Finally, the mechanism allows the detection of the last predictor, as opposed to Sutton and Barto rules (see section 2.2) that detect the earliest. This is important since spreading calls from a goal to its successive subgoals requires the learning of all intermediate events of a perceptive sequence, and not only the first, even if it is actually predictive of the last. 
Application This mechanism has been used inside each map of a multi-map architecture for robot control (Frezza-Buet and Alexandre 1998b). This architecture contains many maps for detecting multi-modal events that are linked with the mechanism presented here. The multimodal sequences that are learned are the basis for a competition within units in the model. This competition allows the robot to trigger the appropriate action at each time, according to the needs that initiate call activities, according to the perception that initiates excitation activities, and to the knowledge concerning the world stored in the $w_{i j}$. The cortical framework, that drives the design of the map architecture, coupled with the local temporal mechanism described in this section, has led here to an efficient control architecture endowing the robot with the ability to learn elaborated reactive behavior from its experience in the environment.

\subsection{Context detection for bistable transitions}

The previously described model deals with one level sequences (no sub-sequences), providing procedural abilities for reactive behavior. This model refers to posterior cortex functionalities. As we are interested in more complex behaviors, involving planning on the basis of connectionist computation, we are currently studying prefrontal functions. Some early modeling results will be presented here briefly, in order to introduce the use of the context manipulation mechanism detailed here, allowing temporal scheduling of actions.

Prefrontal modeling framework for neural planning As mentioned in section 3, prefrontal functionality is grounded on cortical columns having a bistable activity pattern. The model described now is an attempt to use this ability for planning the behaviour of the robot. Compared with a biological description of the cortex, and more precisely of the prefrontal lobe, our functional approach is of course very rough, but it has to be seen in the context of the design of efficient and highly integrated control architectures. In this modeling framework, prefrontal cortex is a set of units connected to posterior cortex units with one-toone connections (cf. figure 12). The posterior cortex part of the model is similar to the one mentioned in section 4.2. It is a module allowing complex servo-control oriented computation, i.e. a call activity at the level of a posterior unit (the $P_{i}$ s in figure 12) triggers an elaborated action of the robot, as "facing the current target" for example. Then, the role of the prefrontal cortex part of the model (the $F_{i}$ s in figure 12) is to schedule posterior calls, in order to plan the behaviour of the robot towards finding rewarding situations.

This scheduling, described in (Burnod 1989) and in a more computer science oriented way in (Frezza-Buet and Alexandre 1998a), is illustrated by the following example. Let us suppose that a sequence of events $a-b-c$ has to be performed for getting a reward. That means that posterior units $P_{a}, P_{b}$ and $P_{c}$ have to be excited in that order, after successive calls in these three units. The role of associated frontal units $F_{a}, F_{b}$ and $F_{c}$ (cf. figure 12) is then the following. First $F_{a}$ triggers a call on posterior unit $P_{a}$. As a consequence of this call, let us 
suppose that event $e_{a}$ occurs, meaning that $P_{a}$ is excited. Then, $F_{a}$ sends call activity to $F_{b}$, that calls $P_{b}$ to get $e_{b}$ in the same way. When $e_{b}$ occurs, $F_{b}$ transmits the call to $F_{c}$ that enables the occurrence of the rewarded event $e_{c}$. The significance of this sequence, compared with the posterior model of section 4.2, is the way failures of calls are managed. Let us suppose that the call in $P_{a}$ triggered by $F_{a}$ in the previous example is not followed by the event $e_{a}$ that makes $P_{a}$ excited. That means that the current context does not allow the getting of this event, and that something else has to be done before getting $e_{a}$ is possible. What has to be done before is the activation of another sequence of events (they may involve motor events as a consequence of calls), noted $a a-a b$ in figure 12 . The failure of the call in $F_{a}$ makes $F_{a}$ have a sustained specific activity (transition $\mathrm{ON}$ of the bistable) that stacks the purpose of calling $P_{a}$ without calling it anymore. Then, a call is transmitted to $F_{a a}$. When sequence $a a-a b$ has been performed, the perceptive world is supposed to be in a context that allows $e_{a}$ to occur subsequently to a call in $P_{a}$. The detection of this context, which is the temporal mechanism described in this section, triggers the OFF transition of bistable activity in $F_{a}$. The effect of this latter transition is first to stop storing the call that previously failed, and second to retry it, by calling $P_{a}$ again (the call is popped out from the stack). Due to the use of $a a-a b$, this call now allows $e_{a}$ to occur, and $F_{a}$ transmits a call to $F_{b}$ as in the non failing case presented first.

Context detection, which is crucial for the neuronal stacking allowed by the frontal cortex model, has to be robust to the intrinsic asynchronousness of perceptions. This context detection, which is the learning rule for frontal sequences in the model, is presented now and details concerning the use of this mechanism for robot control can be found in (Frezza-Buet and Alexandre 1998a).

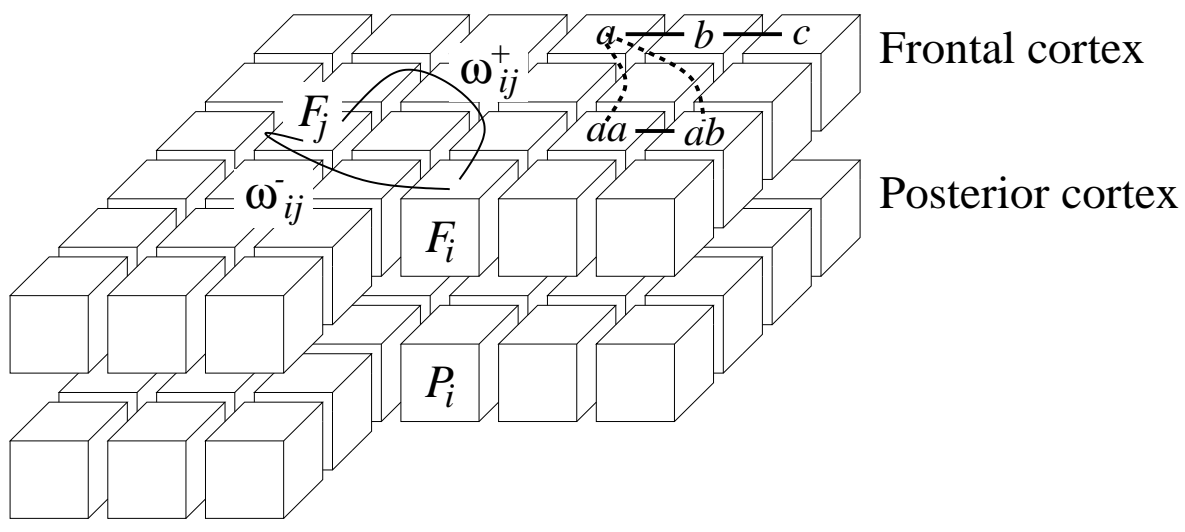

Fig. 12. Prefrontal modeling framework. 
Asynchronous context detection The purpose of the mechanism is to enable a bistable frontal unit $F_{i}$ (cf. figure 12) to learn perceptive context. This context must ensure that a call on the corresponding $P_{i}$ will succeed in getting the associated event $e_{i}$. At the level of $F_{i}$, let us consider two events $e_{i}^{+}$and $e_{i}^{-}$, respectively representing the success of call in $A_{i}$ and its failure $\left(F_{i}\right.$ gives up calling $A_{i}$ ). Learning occurs during both $e_{i}^{+}$and $e_{i}^{-}$, as detailed below.

For any frontal connection between $F_{i}$ and $F_{j}$, a couple of weights $\left(w_{i j}^{+}, w_{i j}^{-}\right)$ are used. Weights $w_{i j}^{+}$and $w_{i j}^{-}$store correlations between excitation in $P_{j}$ and respective events $e_{i}^{+}$and $e_{i}^{-}$. Using these weights, two kinds of contextual activities $\hat{c}_{i}$ and $\tilde{c}_{i}$ are computed, according to equations 22 where operator $[x]^{+}$ returns $x$ if $x>0$ and 0 otherwise.

$$
\begin{aligned}
E_{j} & =f_{\theta}\left(E_{j}^{\mathrm{exci}}\right) \times f_{\theta}\left(E_{j}^{\mathrm{rec}}\right) \\
\omega_{i \max }^{+} & =\max _{j} \omega_{i j}^{+} \\
\tilde{x}_{i}^{+} & =\left(1-\omega_{i \max }\right)+\omega_{i \max } \frac{\sum_{j} \omega_{i j} E_{j}}{\omega_{i j}} \\
\hat{x}_{i}^{+} & =1-\max _{j}\left\{\left(1-E_{j}\right) \omega_{i j}^{+}\right\}_{j} \\
x_{i}^{-} & =\max _{j}\left\{E_{j} \times f_{\rho}^{\prime}\left(\omega_{i j}^{-}-\omega_{i j}^{+}\right)\right\} \\
& \quad \text { where } f_{\rho}^{\prime}= \\
\tilde{c}_{i} & \leftarrow\left[\tilde{x}_{i}^{+}-x_{i}^{-}\right]^{+} \\
\hat{c}_{i} & \leftarrow\left[\hat{x}_{i}^{+}-x_{i}^{-}\right]^{+}
\end{aligned}
$$

The value $E_{j}$ is the trace of excitation activity of $P_{j}$ (intensity of $e_{j}$ modulated by its recency). The value $\tilde{x}_{i}^{+}$is the fitting of the $E_{j}$ distribution to the stored $w_{i j}^{+}$weights. If no strong correlation has been detected between any $E_{j}$ and $e_{i}^{+}\left(\omega_{i \max }^{+}\right.$is weak $)$, the $w_{i j}^{+}$are not significant, the context $\tilde{x}_{i}^{+}$is then good by default (close to 1 ). The context value $\tilde{x}_{i}^{+}$is sensitive to changes in the $E_{j}$ distribution, allowing the detection that the current distribution is going closer to the ideal one (defined by the $w_{i j}^{+}$). As this context is too permissive for reliable context detection, another context value $\hat{x}_{i}^{+}$is defined, which is high when the $E_{j}$ distribution matches exactly the $w_{i j}^{+}$. Finally, if one of the current $E_{j}$ has 
been detected as being correlated to $e_{i}^{-}$without being correlated to $e_{i}^{+}\left(\omega_{i j}^{-}-\omega_{i j}^{+}\right.$ is high), this $E_{j}$ is predictive of the failure of the call, and the $x_{i}^{-}$context value is high. Using the contexts $\tilde{x}_{i}^{+}, \hat{x}_{i}^{+}$and $x_{i}^{-}$, two respectively permissive and not permissive contexts $\tilde{c}_{i}$ and $\hat{c}_{i}$ are defined.

As $\hat{c}_{i}$ is reliable, it is used to trigger the OFF transition of $F_{i}$. On the other hand $\tilde{c}_{i}$, being sensitive to the improvement of the distribution of perception, is used to sustain bistable activity of $F_{i}$ until $\hat{c}_{i}$ allows the OFF transition.

The learning rule for the $\omega i j^{+}$is straightforward, once the previous values are computed (cf. equation 23). Note that the $\omega_{i j}^{-}$are computed only when the call fails but it was supposed to succeed $\left(\hat{c}_{i}>0\right)$.

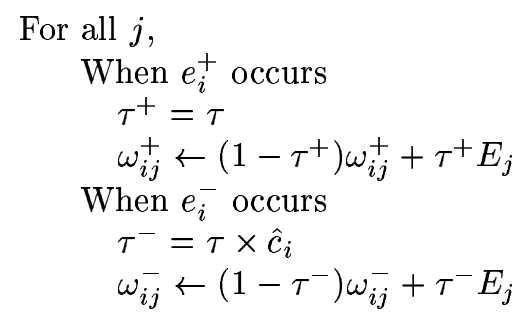

Interest of the context learning mechanism The context detection is robust (Frezza-Buet 1999) to asynchronous perception, due to correlation with traces. It also enables one to separate events $E_{j}$ that are often occurring whenever a call in $P_{i}$ succeeds $\left(e_{i}^{+}\right)$or not $\left(e_{i}^{-}\right)$from the events $E_{j}$ that are responsible for the failure. This mechanism, coupled with stacking properties of bistable activation, is involved in the scheduling of action schemes, synchronizing calls towards the posterior cortex model by defining when a sub-sequence has succeeded. Learned contexts are also used to determine which sub-sequence to call when a call fails. Finally, the same context mechanism has been reused, detecting distribution of call activities among the frontal units, to learn which schemes are not compatible with the execution of others.

\subsection{Discussion}

The design of mechanisms presented in this section is driven by engineering constraints (robustness, integration into an efficient architecture), that lead us not just to stick to the biological background. Using biological inspiration for such purposes is constrained by a trade off between biological validity and computational efficiency. Nevertheless, cortical modeling offers us a framework to develop highly integrated applications. These open architectures can then be refined and extended consistently.

\section{Conclusion}

The main goal of this chapter was to present a cortical framework in which isolated neurobiologically inspired mechanisms can be integrated. It was shown in 
particular that these mechanisms can act at different levels of time, for different kinds of elementary functions. Data from cortical organization and functioning offer a framework for embedding these mechanisms in a way that yields neurobiological plausibility at the neuronal as well as the behavioral level.

As a conclusion, in a schematic way, we can now consider in turn the mechanisms and their time scale, with the corresponding neuronal and behavioral description.

1. The time scale of one millisecond corresponds, at the neuronal level, to the duration of a spike and to the duration of synaptic transmission from one neuron to its closest neighbors. It thus corresponds to the minimal time scale for a bit of information. At the behavioral level, coincidence detectors, in peripheral neural structures, can work at this time scale.

2. The time scale of ten milliseconds corresponds, at the neuronal level in the central structures, to the coding level of spike intervals, since the maximal frequency cannot exceed $100 \mathrm{~Hz}$ in these structures. This thus corresponds to the minimal timing from one area to the next. At the behavioral level, it corresponds to the focus of attention or to the timing of feedback information flow from an higher level to a lower level map.

3. The time scale of one hundred milliseconds corresponds, at the neuronal level, to the activation dynamics of a population of neurons, which are locally synchronized at this time scale. It is the basic timing of activities in the cortex which are linked with simple sensory and motor events. At the behavioral level, it corresponds to the minimal reaction times from the first processing layer (stimulus) to the last (recognition or action). It is thus the minimal time for the simplest sensorimotor loops.

4. The time scale of one second corresponds, at the neuronal level, to the time scale of the basic processes which can result in learning. Neurons in the higher levels of associative cortex can stay active on this time scale, even if the stimulus is no longer present. At the behavioral level, it corresponds to the time scale of correspondences between sensory and motor events on different modalities which can produce reinforcement. It also corresponds to a level of information processing which has strong intrinsic regulations within modalities (for example, the exploration of an object).

5. The time scale of ten seconds corresponds, at the neuronal level, to the typical time scale of working memory in the frontal regions of the cortex. The learning mechanism is performed by the control of bistable states of frontal neurons in order to build stacks. At the behavioral level, this time scale corresponds to the processes in the frontal cortex allowing the organization of temporal aspects of behavior like the exploration of a scene.

6. The time scale of one hundred seconds and more corresponds, at the neuronal level, to the very long time constants of some neuronal intrinsic metabolic and genetic processes. Rhythms with long periods can be produced by such structures as the reticular formation and the hypothalamic nuclei. Such internal clocks can influence cortical activity by modulators which can switch the intrinsic temporal programs of large populations of cortical neurons. At 
the behavioral level, these biological rhythms can have large contextual influence (like emotion) and can produce global regulation of the behavioral programs within the whole network.

On the one hand, each basic temporal mechanism that we have presented above can be related to one (or two consecutive) of these temporal resolutions and to the corresponding neuronal and behavioral mechanisms. On the other hand it is clear that a fully plausible behavioral model should include all of the six levels of time. In any case, it should not be restricted to one or two levels. We believe that the cortical framework that we have presented here allows one to work at the same time at these different temporal levels, with the corresponding behavioral abilities.

\section{References}

Alexandre, F. (1996). Connectionist cognitive processing for invariant pattern recognition. In Proceedings International Conference on Pattern Recognition.

Ans, B., Coiton, Y., Gilhodes, J., and Velay, J. (1994). A neural network model for temporal sequence learning and motor programming. Neural Networks.

Béroule, D. (1990). Guided propagation : current state of theory and application. In Soulié, F. F. and Hérault, J., editors, Neurocomputing. NATO ASI Serie. Springer Verlag Berlin Heidelberg.

Brodmann, K. (1909). Vergleichende Lokalisationslehre der grobhirnrinde. J.A. Barth, Leipzig.

Burnod, Y. (1989). An adaptive neural network the cerebral cortex. Masson.

Dehaene, S. and Changeux, J.-P. (1989). A simple model of prefrontal cortex function in delayed-response task. Journal of Cognitive Neuroscience, 1(3):244261.

Dominey, P., Arbib, M., and Joseph, J.-P. (1995). A model of corticostriatal plasticity for learning oculomotor associations and sequences. Journal of Cognitive Neuroscience, 7(3):311-336.

Durand, S. and Alexandre, F. (1996). TOM, a new temporal neural network architecture for speech signal processing. In Proceedings IEEE International Conference on Acoustics, Speech and Signal Processing, Atlanta.

Elman, J. L. (1990). Finding structure in time. Cognitive Science, 14:179-211.

Frezza-Buet, H. (1999). Un modèle de cortex pour le comportement motivé d'un agent neuromimétique autonome. $\mathrm{PhD}$ thesis, Université Henri Poincaré Nancy I. In french.

Frezza-Buet, H. and Alexandre, F. (1998a). Multimodal sequence learning with a cortically-inspired model. In JCIS98, Association for Intelligent Machinery, volume 2, pages $24-27$. 
Frezza-Buet, H. and Alexandre, F. (1998b). Selection of action with a corticallyinspired model. In Seventh European Workshop on Learning Robots, pages $13-21$.

Frezza-Buet, H. and Alexandre, F. (1999). Specialization within cortical models: An application to causality learning. In Proceedings of the 7th European Symposium on Artificial Neural Networks.

Gerstner, W. (1998). Spiking neurons. In Maass, W. and Bishop, C., editors, Pulsed Neural Networks. Bradford Book, MIT Press.

Gove, A., Grossberg, S., and Mingolla, E. (1995). Brightness perception, illusory contours, and corticogeniculate feedback. Visual Neuroscience, 12:1027-1052.

Grossberg, S. (1984). Some normal and abnormal behavioral syndromes due to transmitter gating of opponent processes. Biological Psychiatry, 19(7):10751117.

Grossberg, S., Mingolla, E., and Williamson, J. (1995). Synthetic aperture radar processing by a multiple scale neural system for boundary and surface representation. Neural Network, 8:1005-1028.

Grossberg, S. and Schmajuk, N. A. (1987). Neural dynamics of attentionally modulated pavlovian conditioning : Conditioned reinforcement, inhibition, and opponent processing. Psychobiology, 15(3):195-240.

Grossberg, S. and Schmajuk, N. A. (1989). Neural dynamics of adaptive timing and temporal discrimination during associative learning. Neural Network, 2:79-102.

Guigon, E. (1993). Modelisation des proprietes du cortex cerebral : Comparaison entre aires visuelles, motrices et préfrontales. $\mathrm{PhD}$ thesis, École centrale de Paris. in English.

Hartwich, E. and Alexandre, F. (1997). A Speech Recognition System using an Auditory Model and TOM Neural Network. In Pearson, O.-W., Steele, N.-C., and Albrecht, R.-F., editors, Proceedings International Conference of Artificial Neural Nets and Genetic Algorithms, Norwich. Springer Verlag.

Littman, M. L. (1996). Algorithms for Sequential Decision Making. PhD thesis, Department of Computer Science at Brown University.

Maass, W. and Bishop, C., editors (1998). Pulsed Neural Networks. MIT Press.

Pavlov, I. P. (1927). Conditioned Reflexes (V.Anrep, trans.). London: Oxford University Press".

Pearlmutter, B. A. (1990). Dynamic recurrent neural networks. Technical Report CMU-CS-90-196, Carnegie Mellon University.

Reiss, M. and Taylor, J. (1991). Storing temporal sequences. Neural Networks, 4:773-787.

Rescorla, W. A. and Wagner, A. R. (1972). Classical conditioning II: Current research and theory, chapter A theory of Pavlovian conditioning: Variations in the effectiveness of reinforcement and non-reinforcement. Black, A.H. and Prokasy, W. F., appleton-century-crofts edition. 
Skinner, B. (1938). The Behavior of Organisms.

Suga, N. (1990). Cortical computational maps for auditory imaging. Neural Networks, 3:3-21.

Sun, R. and Alexandre, F., editors (1997). Connectionist - Symbolic Interpretation; from Unified to Hybrid Approaches. Lawrence Erlbaum Associates.

Sutton, R. S. and Barto, A. G. (1981). Toward a modern theory of adaptative network : Expectation and prediction. Psychological Review, 88(2):135-170.

Waibel, A., Hanazawa, T., Hinton, G., Shikano, K., and Lang, K. (1989). Phoneme recognition using time delay neural networks. In IEEE Transactions on Acoustics Speech and Signal Processing, volume 37.

Watkins, C. J. (1989). Learning from delayed rewards. PhD thesis, University of Cambridge. 